\title{
Exact analytical solution of a time-reversal-invariant topological superconducting wire
}

\author{
Armando A. Aligia $\oplus^{1}$ and Alberto Camjayi ${ }^{2}$ \\ ${ }^{1}$ Centro Atómico Bariloche and Instituto Balseiro, CNEA, 8400 S. C. de Bariloche, Argentina \\ ${ }^{2}$ Departamento de Física, FCEyN, Universidad de Buenos Aires and IFIBA, Pabellón I, Ciudad Universitaria, 1428 Buenos Aires, Argentina
}

(Received 10 May 2019; revised manuscript received 19 July 2019; published 10 September 2019)

\begin{abstract}
We consider a model proposed before for a time-reversal-invariant topological superconductor which contains a hopping term $t$, a chemical potential $\mu$, an extended $s$-wave pairing $\Delta$, and spin-orbit coupling $\lambda$. We show that for $|\Delta|=|\lambda|, \mu=t=0$, the model has an exact analytical solution defining new fermion operators involving nearest-neighbor sites. The many-body ground state is fourfold degenerate due to the existence of two zero-energy modes localized exactly at the first and last sites of the chain. These four states show entanglement in the sense that creating or annihilating a zero-energy mode at the first site is proportional to a similar operation at the last site. By continuity, this property should persist for general parameters. Using these results, we discuss some statements related to the so-called time-reversal anomaly. The addition of a small hopping term $t$ for a chain with an even number of sites breaks the degeneracy, and the ground state becomes unique with an even number of particles. We also consider a small magnetic field $B$ applied to one end of the chain. We compare the many-body excitation energies and spin projection along the spin-orbit direction for both ends of the chains obtained treating $t$ and $B$ as small perturbations with numerical results in a short chain, obtaining good agreement.
\end{abstract}

DOI: $10.1103 /$ PhysRevB.100.115413

\section{INTRODUCTION}

In recent years, there has been a lot of interest in topological superconductors. One of the main reasons for this attention is the existence of Majorana fermions at the ends of one-dimensional wires, which might be used in quantum computation exploiting their non-Abelian nature [1,2].

The first theoretical proposals [3-7] and experimental research [8-12] were focused on systems in which timereversal symmetry is broken. More recently, theoretical research on time-reversal-invariant topological superconductors (TRITOPS) has developed [13-41].

The TRITOPS belong to class DIII in the classification of topological superconductors [42]. As such, they host a zeroenergy fermionic excitation (or, equivalently, a Kramers pair of Majorana fermions) at each end of the wire. This "Majorana Kramers qubit" has been proposed as the basis of a universal gate set for quantum computing [41].

Zhang et al. [18] proposed to construct TRITOPS wires via the proximity effect between nodeless extended $s$-wave iron-based superconductors and semiconducting systems with large Rashba spin-orbit coupling. An extended $s$-wave superconducting gap $\Delta$ and spin-orbit coupling $\lambda$ are the basic ingredients in the TRITOPS physics.

A remarkable property of the TRITOPS wires is that the many-body ground state is characterized by a fractional spin projection along the spin-orbit coupling (which we choose to be $z$ ) at each end of the wire. Specifically, for the left and right ends $S_{\text {left }}^{z}, S_{\text {right }}^{z}= \pm 1 / 4$.

The first argument to show this fractional spin $[19,40]$ was based on the so-called time-reversal anomaly, first proposed for two- and three-dimensional TRITOPS [13]. The argument can be summarized as follows. Denote as $a_{\text {left } \uparrow}$ the annihilation operator of the zero-energy mode at the left end of the chain. It commutes with the Hamiltonian $H$, and $\left[a_{\mathrm{left} \uparrow}, S_{z}\right]=$ $(1 / 2) a_{\mathrm{left} \uparrow}$, where $S_{z}$ is the total spin projection. In addition, as shown below, the time reversal of $a_{\text {left } \uparrow}$ is proportional to $a_{\text {left } \uparrow}^{\dagger}$ :

$$
K a_{\mathrm{left} \uparrow} K^{\dagger}=a_{\mathrm{left} \downarrow} \propto a_{\mathrm{left} \uparrow}^{\dagger},
$$

with $K$ being the time-reversal operator, so there is only one independent fermion at the left end, and the same happens at the right end. Let us assume that $\left|G_{0}\right\rangle$ is one of the degenerate ground states, with $a_{\text {left }}\left|G_{0}\right\rangle=0$. Then, since $\left[a_{\text {left } \uparrow}^{\dagger}, H\right]=0$, also $\left|G_{1}\right\rangle=a_{\text {left } \uparrow}^{\dagger}\left|G_{0}\right\rangle$ belongs to the ground-state manifold (if it does not vanish). Due to timereversal invariance of the Hamiltonian, one might expect that $\left|G_{0}\right\rangle$ and $\left|G_{1}\right\rangle$ are time-reversal partners, and this implies $\left\langle G_{1}\left|S_{\text {left }}^{z}\right| G_{1}\right\rangle=-\left\langle G_{0}\left|S_{\text {left }}^{z}\right| G_{0}\right\rangle$, where $S_{\text {left }}^{z}$ is the spin projection at the left end of the chain [33]. On the other hand, since $a_{\text {left } \uparrow}$ annihilates a spin up (or creates a spin down) at the left of the chain $\left\langle G_{1}\left|S_{\text {left }}^{z}\right| G_{1}\right\rangle-\left\langle G_{0}\left|S_{\text {left }}^{z}\right| G_{0}\right\rangle=-1 / 2$, and then $\left\langle G_{0}\left|S_{\text {left }}^{z}\right| G_{0}\right\rangle=1 / 4$. In this argument, neither the right end nor the whole degeneracy of the ground state is considered. Our results, in which the many-body states are constructed explicitly, shed light on the underlying physics (see Sec. III B 3). We find that $K\left|G_{0}\right\rangle$ is not proportional to $\left|G_{1}\right\rangle$ but coincides with a third ground state.

In a previous publication, the excitations at both ends were studied [33]. The contribution of each site to the operators $a_{\text {left } \sigma}$ and $a_{\text {right } \sigma}$ decays exponentially with the distance to the corresponding end, with a decay length $\lambda_{e}$ determined by solving a quartic equation. For the particular case of the chemical potential $\mu=0$ and the length of the chain $L \rightarrow \infty$, an analytical form of the end operators was given. Formally, one of the many-body states $\left|e_{1}\right\rangle$ that is part of the groundstate manifold for $L \rightarrow \infty$ is constructed in a way similar 
to the ground state of the BCS Hamiltonian, as the product of all annihilation operators $\Gamma_{v}$ satisfying $\left[\Gamma_{v}, H\right]=E_{v} \Gamma_{v}$ with positive $E_{v}$. For finite $L$, an exponentially small mixing of $a_{\text {left } \sigma}$ and $a_{\text {right } \sigma}$ takes place. Two new mixed annihilation operators $\gamma_{\sigma}$ with $\left[\gamma_{\sigma}, H\right]=E_{\sigma} \gamma_{\sigma}$ with positive $E_{\sigma}$ are found, so that the (now nondegenerate) ground state becomes $\left|g_{e}\right\rangle=$ $\gamma_{\uparrow} \gamma_{\downarrow}\left|e_{1}\right\rangle$. Although the explicit form of $\left|e_{1}\right\rangle$ is not known, the facts that it is time reversal invariant in the absence of a magnetic field and that the operators $\Gamma_{v}$ correspond to finite energy have been used to calculate the spin projection $S_{\text {right }}^{z}$ for the ground state and the first excited states with odd number of particles, in particular for a magnetic field applied only to the right end, finding fractional values.

Some open questions regarding the nature of the manybody ground state still remain. For example, for $L \rightarrow \infty$ there are two independent zero-energy modes, one at each end of the chain. Then, one expects a fourfold-degenerate ground state depending on whether the occupation number of these two fermions is 0 or 1 . However, in principle, there are 16 combinations of the operators $\Gamma_{\text {lefto }}$ and $\Gamma_{\text {right } \sigma}$ that could be applied to $\left|e_{1}\right\rangle$. How are they related? To answer this question the explicit form of $\left|e_{1}\right\rangle$ is needed.

In this work, we report on the exact analytical solution of the model for particular parameters $(|\Delta|-|\lambda|=\mu=t=0)$, with finite $L$ and open boundary conditions. This allows us to construct explicitly not only the one-body operators that diagonalize the Hamiltonian but also the four many-body states that are part of the ground state. We find that $\Gamma_{\text {right } \sigma}\left|e_{1}\right\rangle$ is proportional to $\Gamma_{\text {left }}\left|e_{1}\right\rangle$, where $\left|e_{1}\right\rangle$ is constructed as indicated above. By continuity, this property should be valid for general parameters inside the topological phase $(|\mu|<2|\lambda|)$. This indicates that although $\left|e_{1}\right\rangle$ does not seem to contain information about the zero-energy modes (it is constructed with operators that commute with the zero-energy ones), it is, in fact, an entangled state and its ends are related.

Using perturbative methods, we also discuss the effect of a small hopping $t$ and a magnetic field applied to one end on the exact solution. The analytical results for the many-body states are supported by numerical diagonalization of small systems.

This paper is organized as follows. In Sec. II the model is described. In Sec. III we construct the exact analytical solution of the model for $|\Delta|-|\lambda|=\mu=t=0$ and describe the many-body ground state. We also calculate the expectation values of the spin projection at the ends for the different states that compose the ground state or appropriate linear combinations of them, finding the fractional values $\pm 1 / 4$ expected from previous works. In Sec. IV we analyze perturbatively the effect of a small hopping $t$ on the exact solution and compare this analysis with numerical results. In Sec. V we present a summary and a brief discussion.

\section{MODEL}

The Hamiltonian describing the system reads $[18,30]$

$$
H=\sum_{\sigma}\left\{\sum_{j=1}^{L-1}\left(-t c_{j+1 \sigma}^{\dagger} c_{j \sigma}+i s_{\sigma} \lambda c_{j+1 \sigma}^{\dagger} c_{j \sigma}+s_{\sigma} \Delta e^{i \phi} c_{j+1 \sigma}^{\dagger} c_{j \bar{\sigma}}^{\dagger}+\text { H.c. }\right)-\mu \sum_{j=1}^{L} c_{j \sigma}^{\dagger} c_{j \sigma}\right\},
$$

where $s_{\uparrow, \downarrow}= \pm 1$ and $\bar{\uparrow}=\downarrow, \bar{\downarrow}=\uparrow$. The parameter $t$ corresponds to the nearest-neighbor hopping, $\mu$ is the chemical potential, $\lambda$ is the Rashba spin-orbit coupling, and $\Delta$ is the strength of the extended $s$-wave pairing.

In the following we will take for simplicity $\Delta=\lambda=1$, $\phi=t=\mu=0$. The formalism used can be changed in a straightforward way to include a finite phase $\phi$ and other signs of $\Delta$ and $\lambda$. The effect of finite $t$ is treated in Sec. IV, and the general case is discussed in Sec. V.

For $\phi=0$, the Hamiltonian is invariant under time-reversal symmetry. In addition, the Hamiltonian conserves parity and the total spin projection $S_{z}$ (the total spin in the direction of the Rashba spin-orbit coupling). Below we will use these three symmetries.

\section{CONSTRUCTION OF THE EXACT ANALYTICAL SOLUTION}

\section{A. One-particle operators}

In this section, we look for annihilation operators $\Gamma_{v}$, satisfying

$$
\left[\Gamma_{v}, H\right]=E_{v} \Gamma_{v},
$$

that diagonalize the Hamiltonian (2) for $\Delta=\lambda=1, \phi=$ $t=\mu=0$. Since Eq. (3) implies that $\Gamma_{v}^{\dagger}$ satisfies the same equation with the opposite sign of $E_{v}$, we can redefine the operators so that $E_{v} \geqslant 0$, permuting $\Gamma_{v}$ and $\Gamma_{v}^{\dagger}$ if necessary.

We define the following operators:

$$
\begin{aligned}
& a_{j \sigma}=\left(c_{j \sigma}+i c_{j \bar{\sigma}}^{\dagger}\right) / \sqrt{2}, \\
& b_{j \sigma}=\left(c_{j \sigma}-i c_{j \bar{\sigma}}^{\dagger}\right) / \sqrt{2},
\end{aligned}
$$

with the property

$$
a_{j \sigma}^{\dagger}=-i a_{j \bar{\sigma}}, b_{j \sigma}^{\dagger}=i b_{j \bar{\sigma}} .
$$

Using Eq. (2), the following commutators can be obtained for a finite chain of $L$ sites:

$$
\begin{aligned}
& {\left[a_{j \sigma}, H\right]= \begin{cases}2 s_{\sigma} i b_{j-1 \sigma} & \text { if } j>1, \\
0 & \text { otherwise, }\end{cases} } \\
& {\left[b_{j \sigma}, H\right]= \begin{cases}-2 s_{\sigma} i a_{j+1 \sigma} & \text { if } j<L, \\
0 & \text { otherwise, }\end{cases} }
\end{aligned}
$$

with $j=1, \ldots, L$.

Clearly, $a_{1 \sigma}$ and $b_{L \sigma}$, taking into account Eqs. (5), correspond to the zero-energy modes, one for each end of the chain, expected from the topological character of the TRITOPS phase $[18,30]$. The remaining operators mix nearest-neighbor sites as sketched in Fig. 1, resembling the exact analytical solution of the Kitaev model [3]. 


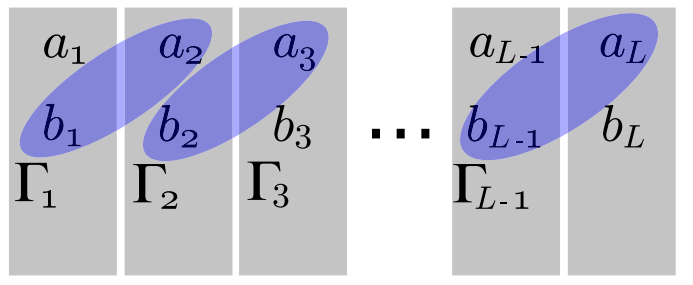

FIG. 1. Sketch of the construction of the annihilation operators of finite energy, leaving the zero-energy modes $a_{1 \sigma}$ and $b_{L \sigma}$ at the ends.

Defining for $j<L$ the operators

$$
\Gamma_{j \sigma}=\left(b_{j \sigma}-i s_{\sigma} a_{j+1 \sigma}\right) / \sqrt{2},
$$

it is easy to see, using Eqs. (6), that their commutators with the Hamiltonian are

$$
\left[\Gamma_{j \sigma}, H\right]=2 \Gamma_{j \sigma} .
$$

Therefore, the desired annihilation operators are obtained.

Note that, under time reversal $K$, the operators defined so far transform as

$$
K \alpha_{j \uparrow} K^{\dagger}=\alpha_{j \downarrow}, K \alpha_{j \downarrow} K^{\dagger}=-\alpha_{j \uparrow},
$$

where $\alpha=a, b$, or $\Gamma$.

\section{B. Many-body low-energy eigenstates}

Since there are two independent fermionic modes with zero energy, one at each end of the chain, one expects a fourfolddegenerate ground state depending on the occupancy of these modes. The analysis below as well as many-body calculations in chains with up to $L=6$ sites confirms this expectation.

One of these four states, with an even number of particles, is obtained by applying all annihilation operators with positive energy [those entering Eq. (8)] to the vacuum $|0\rangle$ of the $c_{j \sigma}$ :

$$
\left|e_{1}\right\rangle=N_{L} \prod_{j=1}^{L-1} \Gamma_{j \uparrow} \Gamma_{j \downarrow}|0\rangle,
$$

where $N_{L}$ is a normalization factor. This state is invariant under time reversal [using Eqs. (9), one easily proves that $\left.K\left|e_{1}\right\rangle=\left|e_{1}\right\rangle\right]$ and also $S_{z}\left|e_{1}\right\rangle=0$, where $S_{z}=\sum_{j=1}^{L} S_{j}^{z}$ is the total spin projection. Note that neither the Hamiltonian nor this state has inversion symmetry.

Two states with an odd parity number can be written as

$$
|o \sigma\rangle=N_{o} a_{1 \bar{\sigma}}\left|e_{1}\right\rangle,
$$

where the normalization factor $N_{o}=\sqrt{2}$, as shown below Eq. (23). These states are also eigenstates of the total spin projection with $S_{z}|\sigma \sigma\rangle=\left(s_{\sigma} / 2\right)|o \sigma\rangle$.

Finally, there is another even-state invariant under time reversal and with zero spin projection,

$$
\left|e_{2}\right\rangle=\left(a_{1 \uparrow} a_{1 \downarrow}-a_{1 \downarrow} a_{1 \uparrow}\right)\left|e_{1}\right\rangle .
$$

Using Eqs. (5), it is easy to see that this state is normalized $\left(\left\langle e_{2} \mid e_{2}\right\rangle=1\right)$ and orthogonal to $\left|e_{1}\right\rangle$. In fact, except for normalization factors, $\left\langle e_{1} \mid e_{2}\right\rangle \sim i(\langle o \uparrow \mid o \uparrow\rangle-\langle o \downarrow \mid o \downarrow\rangle)=$ 0 . Therefore, all these states constitute an orthonormal basis of the ground-state manifold.
It might seem surprising that acting with the zero-mode operators at the right end, $b_{L \sigma}$ on $\left|e_{1}\right\rangle$, does not lead to new states that are part of the ground state. Instead, we find

$$
\begin{aligned}
& b_{L \sigma}\left|e_{1}\right\rangle=-\left(s_{\sigma} i\right)^{L-1} a_{1 \sigma}\left|e_{1}\right\rangle, \\
& a_{1 \sigma}\left|e_{1}\right\rangle=-\left(s_{\bar{\sigma}} i\right)^{L-1} b_{L \sigma}\left|e_{1}\right\rangle .
\end{aligned}
$$

This can be proved by induction. For the simplest chain with $L=2$, the ground-state manifold is

$$
\begin{aligned}
& \left|e_{1}\right\rangle=\frac{1}{2}\left[c_{1 \uparrow}^{\dagger} c_{1 \downarrow}^{\dagger}+c_{2 \uparrow}^{\dagger} c_{2 \downarrow}^{\dagger}+i c_{1 \uparrow}^{\dagger} c_{2 \downarrow}^{\dagger}+i c_{1 \downarrow}^{\dagger} c_{2 \uparrow}^{\dagger}\right]|0\rangle, \\
& \left.\left|e_{2}\right\rangle=-\frac{1}{2}\left[1-c_{1 \uparrow}^{\dagger} c_{2 \downarrow}^{\dagger}+c_{1 \downarrow}^{\dagger} c_{2 \uparrow}^{\dagger}-c_{1 \uparrow}^{\dagger} c_{1 \downarrow}^{\dagger} c_{2 \uparrow}^{\dagger} c_{2 \downarrow}^{\dagger}\right]\right]|0\rangle, \\
& |o \downarrow\rangle=\frac{1}{2}\left[c_{1 \downarrow}^{\dagger}+i c_{2 \downarrow}^{\dagger}+i c_{1 \downarrow}^{\dagger} c_{2 \uparrow}^{\dagger} c_{2 \downarrow}^{\dagger}+c_{1 \uparrow}^{\dagger} c_{1 \downarrow}^{\dagger} c_{2 \downarrow}^{\dagger}\right]|0\rangle, \\
& |o \uparrow\rangle=K|o \downarrow\rangle .
\end{aligned}
$$

Using these expressions, it is easy to check that Eq. (13) is valid for $L=2$.

Now we prove its validity for a chain of $L+1$ sites assuming that Eq. (13) holds for $L$ sites.

For a chain of $L+1$ sites, Eq. (10) can be written in the form

$$
\begin{aligned}
\left|e_{1}(L+1)\right\rangle & =N_{L+1} \hat{O}(L+1)|0\rangle, \\
\hat{O}(L) & =\prod_{j=1}^{L-1} \Gamma_{j \uparrow} \Gamma_{j \downarrow} .
\end{aligned}
$$

Then using anticommutation rules and Eq. (13),

$$
\begin{aligned}
a_{1 \uparrow}\left|e_{1}(L+1)\right\rangle & =N_{L+1} \Gamma_{L \uparrow} \Gamma_{L \downarrow} a_{1 \uparrow} \hat{O}(L)|0\rangle \\
& =-(-i)^{L-1} N_{L+1} \Gamma_{L \uparrow} \Gamma_{L \downarrow} b_{L \uparrow} \hat{O}(L)|0\rangle \\
& =(-i)^{L-1} N_{L+1} \hat{O}(L) \Gamma_{L \downarrow} \Gamma_{L \uparrow} b_{L \uparrow}|0\rangle .
\end{aligned}
$$

From the definitions (4) and (7) we find

$$
\Gamma_{L \uparrow} b_{L \uparrow}|0\rangle=i b_{L+1 \uparrow} \Gamma_{L \uparrow}|0\rangle=\frac{-i}{2 \sqrt{2}} c_{L+1 \downarrow}^{\dagger} c_{L \downarrow}^{\dagger}|0\rangle,
$$

and inserting this equation in Eq. (16)

$$
\begin{aligned}
a_{1 \uparrow}\left|e_{1}(L+1)\right\rangle & =-(-i)^{L} N_{L+1} \hat{O}(L) \Gamma_{L \downarrow} b_{L+1 \uparrow} \Gamma_{L \uparrow}|0\rangle \\
& =-(-i)^{L} N_{L+1} b_{L+1 \uparrow} \Gamma_{L \uparrow} \Gamma_{L \downarrow} \hat{O}(L)|0\rangle \\
& =-(-i)^{L} b_{L+1 \uparrow}\left|e_{1}(L+1)\right\rangle,
\end{aligned}
$$

in agreement with Eq. (13). The corresponding relation for the opposite spin of the end operators is obtained using the time-reversal operator $K$.

\section{Ground-state energy}

The energy of the fourfold-degenerate ground state $E_{g}$ can be obtained from the following argument. Let us define the charge conjugation (or electron-hole transformation) $C$ as the one which permutes annihilation and creation operators

$$
C c_{j \sigma}^{\dagger} C=c_{j \sigma}, \quad C c_{j \sigma} C=c_{j \sigma}^{\dagger} .
$$
as

Clearly, $C^{2}=1$. For $\phi=0$, the Hamiltonian (2) transforms

$$
C H C=-2 \mu L-H .
$$


In the case we are considering, with $\mu=0$, this implies that if a many-body state $|i\rangle$ is an eigenstate with energy $E_{i}$, its electron-hole partner $C|i\rangle$ is also an eigenstate with energy $-E_{i}$. Thus, the spectrum is symmetric around zero energy.

Since the system is noninteracting, the excited states are obtained by applying creation operators $\Gamma_{j \uparrow}^{\dagger}$ (each with an energy cost of 2) and zero-energy operators (without energy cost) to $\left|e_{1}\right\rangle$. Clearly, one of the states of highest energy $E_{\max }$ is $\prod_{j=1}^{L-1} \Gamma_{j \uparrow}^{\dagger} \Gamma_{j \downarrow}^{\dagger}\left|e_{1}\right\rangle$, and $E_{\max }-E_{g}=4(L-1)$. In addition, since the total spectrum is symmetric, $E_{\max }+E_{g}=0$. Thus, the ground-state energy is

$$
E_{g}=-2(L-1) \text {. }
$$

This has been confirmed by numerical calculations in small systems.

\section{Expectation value of the spin projection at both ends}

Proceeding in a way similar to that shown above, it is possible to write the state $|o \downarrow\rangle$ (except for a phase) as

$$
\begin{aligned}
b_{L \uparrow}\left|e_{1}(L)\right\rangle= & -N_{L} \hat{O}(L-1) \Gamma_{L-1 \downarrow} b_{L \uparrow} \Gamma_{L-1 \uparrow}|0\rangle \\
= & \frac{-1}{4 \sqrt{2}} N_{L} \hat{O}(L-1)\left[-c_{L \downarrow}^{\dagger}+i c_{L-1 \downarrow}^{\dagger}\right. \\
& \left.+i c_{L-1 \uparrow}^{\dagger} c_{L-1 \downarrow}^{\dagger} c_{L \downarrow}^{\dagger}+c_{L-1 \downarrow}^{\dagger} c_{L \uparrow}^{\dagger} c_{L \downarrow}^{\dagger}\right]|0\rangle .
\end{aligned}
$$

From here it is easy to see that the expectation value of the spin projection at the right end [for the parameters of the exact analytical solution, $\left.S_{\text {right }}^{z}=S_{L}^{z}=\left(c_{L \uparrow}^{\dagger} c_{L \uparrow}-c_{L \downarrow}^{\dagger} c_{L \downarrow}\right) / 2\right]$ becomes

$$
\left\langle o \downarrow\left|S_{\text {right }}^{z}\right| o \downarrow\right\rangle=-1 / 4
$$

since half of the terms of Eq. (22) contribute with $-1 / 2$ and the other half do not contribute. In addition, comparing the norm of $b_{L \uparrow} \Gamma_{L-1 \uparrow} \Gamma_{L-1 \downarrow}|0\rangle$ and $\Gamma_{L-1 \uparrow} \Gamma_{L-1 \downarrow}|0\rangle$ and using Eq. (13), one realizes that the norm entering Eq. (11) is $N_{o}=$ $\sqrt{2}$.

Similarly, with $S_{\text {left }}^{z}=S_{1}^{z}$,

$$
\begin{aligned}
& \left\langle o \downarrow\left|S_{\text {left }}^{z}\right| o \downarrow\right\rangle=\left\langle o \downarrow\left|S_{\text {right }}^{z}\right| o \downarrow\right\rangle=-1 / 4, \\
& \left\langle o \uparrow\left|S_{\text {left }}^{z}\right| o \uparrow\right\rangle=\left\langle o \uparrow\left|S_{\text {right }}^{z}\right| o \uparrow\right\rangle=1 / 4,
\end{aligned}
$$

in agreement with previous results derived for the general case [33]. These results might be expected from Eq. (13). In fact, $S_{z}\left|e_{1}\right\rangle=0$, and $S_{z}|o \downarrow\rangle=S_{z} \sqrt{2} a_{1 \uparrow}\left|e_{1}\right\rangle=-\frac{1}{2}|o \downarrow\rangle$, as can be easily shown using $\left[S_{z}, a_{1 \uparrow}\right]=-a_{1 \uparrow} / 2$. Then $a_{1 \uparrow}$ changes the total spin projection by $-1 / 2$, and since it is a local operator, one would naively expect that this change is concentrated to the left of the chain. However, since the application of a zeromode operator with the same spin projection at the left or right end of the chain has the same physical effect, the change in the total spin is actually equally shared by both ends. A similar argument can be applied for $|o \uparrow\rangle$.

Concerning the expectation values of $S_{\text {left }}^{z}$ and $S_{\text {right }}^{z}$ for states $\left|e_{1}\right\rangle$ and $\left|e_{2}\right\rangle$, they are zero since both states are time reversal invariant. However, this is no longer true for an arbitrary linear combination. Using $K\left|e_{i}\right\rangle=\left|e_{i}\right\rangle \quad(i=1,2)$, $K S_{j}^{z} K^{\dagger}=-S_{j}^{z}(j=1, \ldots, L)$, and the antiunitary properties of $K$, we have

$$
\begin{aligned}
\left\langle e_{i}\left|S_{j}^{z}\right| e_{k}\right\rangle & =\left\langle e_{i}\right|\left(K^{\dagger} K S_{j}^{z} K^{\dagger} K\left|e_{k}\right\rangle\right. \\
& =\overline{\left.\left\langle e_{i}\right| K^{\dagger}\right)\left(K S_{j}^{z} K^{\dagger} K\left|e_{k}\right\rangle\right.}=-\overline{\left\langle e_{i}\left|S_{j}^{z}\right| e_{k}\right\rangle} \\
& =-\left\langle e_{k}\left|S_{j}^{z}\right| e_{i}\right\rangle,
\end{aligned}
$$

where the parentheses separate the operators acting to the right and to the left of the parentheses. Clearly, $\left\langle e_{i}\left|S_{j}^{z}\right| e_{i}\right\rangle=0$, and $\left\langle e_{k}\left|S_{j}^{z}\right| e_{i}\right\rangle$, for $i \neq k$ is a purely imaginary number. It is easy to see that the absolute value of the expectation value of the spin at any site (in particular at the ends $j=1, L$ ) is maximized by the following linear combinations:

$$
\left|e_{3}\right\rangle=\frac{1}{\sqrt{2}}\left(\left|e_{1}\right\rangle+i\left|e_{2}\right\rangle\right), \quad\left|e_{4}\right\rangle=\frac{1}{\sqrt{2}}\left(\left|e_{1}\right\rangle-i\left|e_{2}\right\rangle\right),
$$

with

$$
\left\langle e_{3(4)}\left|S_{j}^{z}\right| e_{3(4)}\right\rangle= \pm i\left\langle e_{1}\left|S_{j}^{z}\right| e_{2}\right\rangle .
$$

Using Eqs. (5), (12), (11), and (24) and $\left[S_{1}^{z}, a_{1 \uparrow}^{\dagger}\right]=a_{1 \uparrow}^{\dagger} / 2$,

$$
\begin{aligned}
\left\langle e_{1}\left|S_{1}^{z}\right| e_{2}\right\rangle & =\left\langle e_{1}\left|S_{1}^{z}\left(a_{1 \uparrow} a_{1 \downarrow}-a_{1 \downarrow} a_{1 \uparrow}\right)\right| e_{1}\right\rangle \\
& =i\left\langle e_{1}\left|S_{1}^{z}\left(1-2 a_{1 \uparrow}^{\dagger} a_{1 \uparrow}\right)\right| e_{1}\right\rangle \\
& =-i\left\langle e_{1}\left|a_{1 \uparrow}^{\dagger}\left(2 S_{1}^{z}+1\right) a_{1 \uparrow}\right| e_{1}\right\rangle \\
& =\frac{-i}{2}\left\langle o \downarrow\left|\left(2 S_{1}^{z}+1\right)\right| o \downarrow\right\rangle=\frac{-i}{4} .
\end{aligned}
$$

This result is easily verified for $L=2$ using Eqs. (14).

Inserting Eq. (28) in Eq. (27) and using $\left\langle e_{i}\left|S_{j}^{z}\right| e_{i}\right\rangle=0$ for $j \neq 1$ or $L$, we finally obtain

$$
\begin{aligned}
& \left\langle e_{3}\left|S_{\text {left }}^{z}\right| e_{3}\right\rangle=-\left\langle e_{3}\left|S_{\text {right }}^{z}\right| e_{3}\right\rangle=1 / 4, \\
& \left\langle e_{4}\left|S_{\text {left }}^{z}\right| e_{4}\right\rangle=-\left\langle e_{4}\left|S_{\text {right }}^{z}\right| e_{4}\right\rangle=-1 / 4 .
\end{aligned}
$$

From these equations, it is clear that application of a magnetic field in the direction of the spin-orbit coupling $z$ applied at only one of the ends breaks the degeneracy of the ground state, favoring one of the states $\left|e_{3}\right\rangle$ and $\left|e_{4}\right\rangle$. The state with lower energy depends on the direction of the field and the end at which it is applied. This point is discussed further in Sec. IV B.

\section{Discussion of the time-reversal anomaly}

The explicit construction of the many-body eigenstates allows us to discuss in detail the arguments involved in the so-called time-reversal anomaly $[13,19,40]$.

As discussed in Sec. I, we can identify an independent zero-mode operator at the left end of the chain $a_{1 \uparrow}=i a_{1 \downarrow}^{\dagger}$, where we have used Eq. (5) in the equality. For the ground state $\left|G_{0}\right\rangle$, with the property $a_{1 \uparrow}\left|G_{0}\right\rangle=0,\left|G_{0}\right\rangle=|o \downarrow\rangle$ can be chosen [see Eqs. (10), (11), and (12)].

A local fermion parity operator is defined as $P_{\text {left }}=$ $2 a_{1 \uparrow}^{\dagger} a_{1 \uparrow}-1$. Clearly, $P_{\text {left }}\left|G_{0}\right\rangle=-\left|G_{0}\right\rangle$. It has been shown that $P_{\text {left }}$ anticommutes with the time-reversal operator $K$ $[13,19,40]$. In the present case, this is proved using Eqs. (5) and (9). This implies that the time-reversal partner of $\left|G_{0}\right\rangle$ ( $|o \uparrow\rangle)$ is even under $P_{\text {left }}$,

$$
P_{\text {left }} K\left|G_{0}\right\rangle=-K P_{\text {left }}\left|G_{0}\right\rangle=K\left|G_{0}\right\rangle .
$$




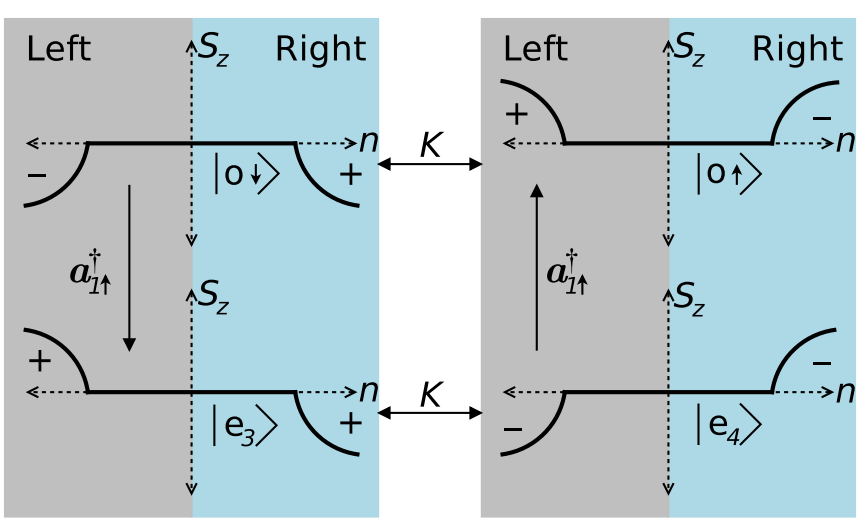

FIG. 2. Many-body ground-state manifold. For the four orthonormal many-body ground states, we sketch the $S_{z}$ value at each chain site $n$. The local parity $\left(P_{\text {left }}, P_{\text {right }}\right)$ value is indicated with $\mathrm{a}+$ or - at the corresponding end. The states are accommodated such that left and right panels are related by time-reversal symmetry and top and bottom states are connected by the action of $a_{1 \uparrow}^{\dagger}$.

Since time reversal should clearly commute with the total number of fermions in the system, the fact that $\left\{K, P_{\text {left }}\right\}=0$ (note that one might expect that in a semi-infinite chain, $P_{\text {left }}$ is the total fermion parity of the system) is known as the timereversal anomaly. To resolve this apparent contradiction, the other end of the chain must be considered. Defining the corresponding local operator on the right $P_{\text {right }}=-\left(2 b_{L \uparrow}^{\dagger} b_{L \uparrow}-1\right)$ (as in Ref. [17]), the product $P=P_{\text {left }} P_{\text {right }}$ coincides with the total fermion parity, and while $\left\{K, P_{\text {left }}\right\}=\left\{K, P_{\text {right }}\right\}=$ $0,[K, P]=0$. This is in complete agreement with previous arguments $[13,19,40]$.

However, for systems which conserve one component of the total spin ( $S_{z}$ in our case), an additional argument, related to the time-reversal anomaly, is usually invoked to explain the spin expectation value at the end of the chain [40]. Let $\left|G_{0}\right\rangle$ be one of the degenerate ground states with $a_{1 \uparrow}\left|G_{0}\right\rangle=0$. Then also $\left|G_{1}\right\rangle=a_{1 \uparrow}^{\dagger}\left|G_{0}\right\rangle$ belongs to the ground-state manifold. On the other hand, since $a_{1 \uparrow}$ annihilates a spin up (or creates a spin down), to the left of the chain $\left\langle G_{1}\left|S_{\text {left }}^{z}\right| G_{1}\right\rangle-$ $\left\langle G_{0}\left|S_{\text {left }}^{z}\right| G_{0}\right\rangle=-1 / 2$. Due to time-reversal invariance of the Hamiltonian, one might expect that $\left|G_{0}\right\rangle$ and $\left|G_{1}\right\rangle$ are timereversal partners, $\left\langle G_{1}\left|S_{\text {left }}^{z}\right| G_{1}\right\rangle=-\left\langle G_{0}\left|S_{\text {left }}^{z}\right| G_{0}\right\rangle$, and then $\left\langle G_{0}\left|S_{\text {left }}^{z}\right| G_{0}\right\rangle=1 / 4$.

The incorrect assumption in the argument discussed above is to associate the ground state $\left|G_{1}\right\rangle$ with the time-reversal partner of $\left|G_{0}\right\rangle$. In fact, as shown previously, it is possible to choose $\left|G_{0}\right\rangle=|o \downarrow\rangle$. Algebraic manipulations similar to those used in Sec. III B 2 lead to $\left|G_{1}\right\rangle=\left|e_{3}\right\rangle$, given by the first equation in Eq. (26). This is because the ground state is fourfold degenerate and contains more states than just $\left|G_{0}\right\rangle$ and $K\left|G_{0}\right\rangle=|o \uparrow\rangle$.

Another possible choice of $\left|G_{0}\right\rangle$ (see Fig. 2), with the property $a_{1 \uparrow}\left|G_{0}\right\rangle=0$, is $\left|e_{4}\right\rangle$ given by the second equation in Eq. (26). In this case $K\left|G_{0}\right\rangle=\left|e_{3}\right\rangle$, which is also orthogonal to $\left|G_{1}\right\rangle=a_{1 \uparrow}^{\dagger}\left|G_{0}\right\rangle$. An important difference between the choices $|o \downarrow\rangle$ and $\left|e_{4}\right\rangle$ is that $K^{2}|o \downarrow\rangle=-|o \downarrow\rangle\left(K^{2}\left|e_{4}\right\rangle=\right.$ $\left.\left|e_{4}\right\rangle\right)$, as expected for a system with an odd (even) number of particles. Note that for both possible choices of $\left|G_{0}\right\rangle,\left|G_{0}\right\rangle$ and $\left|G_{1}\right\rangle$ have opposite expectation values of $S_{\text {left }}^{z}$ (see Fig. 2) but the same value of $S_{\text {right }}^{z}$, as shown in Sec. III B 2. This is a physical indication that $\left|G_{0}\right\rangle$ and $\left|G_{1}\right\rangle$ cannot be time-reversal partners.

Another shortcoming in the argument is that $a_{1 \uparrow}^{\dagger}$, being a local operator, should change the expectation value of $S_{\text {left }}^{z}$ (not only the total $S_{z}$ ) by $1 / 2$; that is, the support of $a_{1 \uparrow}^{\dagger}$ is near the boundary. This is, in fact, true when $a_{1 \uparrow}^{\dagger}$ is applied to $\left|G_{0}\right\rangle=$ $|o \downarrow\rangle$ or $\left|e_{4}\right\rangle$ but not in general. An example is when it is applied to $\left|e_{1}\right\rangle$ since, rather surprisingly, $a_{1 \uparrow}^{\dagger}\left|e_{1}\right\rangle \propto b_{L \uparrow}^{\dagger}\left|e_{1}\right\rangle \propto$ $|o \uparrow\rangle$, implying that while $\left\langle e_{1}\left|S_{\text {left }}^{z}\right| e_{1}\right\rangle=\left\langle e_{1}\left|S_{\text {right }}^{z}\right| e_{1}\right\rangle=0$, one has $\left\langle o \uparrow\left|S_{\text {left }}^{z}\right| o \uparrow\right\rangle=\left\langle o \uparrow\left|S_{\text {right }}^{z}\right| o \uparrow\right\rangle=1 / 4$, so that the spin is actually distributed among both chain ends (see Fig. 2).

The fact that the property $\left\langle G_{1}\left|S_{\text {left }}^{z}\right| G_{1}\right\rangle=-\left\langle G_{0}\left|S_{\text {left }}^{z}\right| G_{0}\right\rangle$ still holds true even when they are not time-reversal partners as is normally argued is the reason why the time-reversal anomaly is useful to guess the fractional spin at the chain boundaries. This relation, however, is not due to a symmetry connecting the states but a particularity of the ground-state manifold.

Previous uses of $\left\{K, P_{\text {left }}\right\}=0$ include Josephson junctions with topological superconductors. Inserting the chain in a ring with a magnetic flux, one expects a circulating current in the system. The flux breaks the time-reversal symmetry except for particular values, including zero. As argued first in Ref. [17] for a TRITOPS with a Hamiltonian different from ours, the time-reversal symmetry is spontaneously broken at zero flux for an odd total fermion parity $P$, leading to a spontaneous circulating current, while this does not happen for even parity (see Eqs. (7) of Ref. [17]). While an argument similar to the above-mentioned time-reversal anomaly has been invoked to show this fact, it can also be derived from more general arguments since the states with odd fermion parity have $K^{2}=$ -1 , and therefore, for any such state $|o\rangle, K|o\rangle \neq c|o\rangle$ for any number $c$. Instead, for even parity one can construct states such as $K|e\rangle=|e\rangle$. The ground states of our system [see Eqs. (10), (11), and (12) and see Eqs. (14) for the particular case $L=2$ ] are clear examples. Using $K|e\rangle=|e\rangle$, since the current operator $I$ is odd under time reversal, one can prove that $\langle e|I| e\rangle=0$, following an argument similar to that leading to Eq. (25).

Note that the properties of the ground states under the discrete symmetries of time reversal, total fermion parity, and local fermion parities, although demonstrated for particular parameters, are valid by continuity inside the whole topological phase for $L \rightarrow \infty$. For finite chains, in general, there is a (very small) mixing of the end zero modes that split the ground state, as described in the next section.

\section{EFFECT OF A SMALL HOPPING TERM}

In this section we discuss the effect of the hopping term $H_{t}=-t \sum_{j=1}^{L-1} \sum_{\sigma}\left(c_{j+1 \sigma}^{\dagger} c_{j \sigma}+\right.$ H.c. $)$ on the exact analytical solution. To simplify the analysis we assume $t$ is much smaller than the other energy scales $(t \ll 1)$. However, the main results concerning the splitting of the degeneracy of the many-body states are general for a long enough chain in the topological phase, as discussed below. 


\section{A. One-body operators}

For $L \geqslant 4$, the commutators with the hopping term of the operators defined in Sec. III A satisfying Eq. (3) for $t=0$ are

$$
\begin{aligned}
& {\left[a_{1 \uparrow}, H_{t}\right]=\frac{t}{\sqrt{2}}\left(-\Gamma_{2 \uparrow}+i \Gamma_{2 \downarrow}^{\dagger}\right),} \\
& {\left[b_{L \uparrow}, H_{t}\right]=\frac{t}{\sqrt{2}}\left(-i \Gamma_{L-2 \uparrow}+\Gamma_{L-2 \downarrow}^{\dagger}\right),} \\
& {\left[\Gamma_{j \uparrow}, H_{t}\right]=t \Gamma_{j \downarrow}^{\dagger}+\frac{t}{2}\left(L_{j}+R_{j}\right),}
\end{aligned}
$$

with

$$
\begin{gathered}
L_{j}=\left\{\begin{array}{lr}
0 & \text { if } j=1, \\
-\sqrt{2} a_{1 \uparrow} & \text { if } j=2, \\
-i \Gamma_{j-2 \uparrow}+\Gamma_{j-2 \downarrow}^{\dagger} & \text { if } j>2,
\end{array}\right. \\
R_{j}= \begin{cases}i \Gamma_{j+2 \uparrow}+\Gamma_{j+2 \downarrow}^{\dagger} & \text { if } j<L-2, \\
i \sqrt{2} b_{L \uparrow} & \text { if } j=L-2, \\
0 & \text { if } j=L-1 .\end{cases}
\end{gathered}
$$

The corresponding relations for the operators with opposite spins are obtained by applying the time-reversal operator [see Eqs. (9)].

In first-order perturbation theory, $H_{t}$ lifts the degeneracy of $\Gamma_{j \sigma}$, introducing a hopping to next-nearest neighbors. The subspace of the operators with even $j$ remains decoupled from the corresponding one for odd $j$. For each subspace of operators, diagonalization of $H_{t}$ is equivalent to solving an open chain with $M$ sites and nearest-neighbor hopping. For odd $L, M=(L-1) / 2$ for both subspaces. For even $L$, $M=L / 2-1$ for the sites with even $j$, and $M=L / 2$ for the subspace of $\Gamma_{j \sigma}$ with odd $j$.

By solving these equivalent problems, for arbitrary $L$, we can define states such that

$$
\left[\Gamma_{k \sigma}, H\right]=(2+t \cos k) \Gamma_{k \sigma} .
$$

In the subspace of the operators with odd $j$ they have the form

$$
\Gamma_{k \uparrow}^{o}=\sqrt{\frac{2}{M+1}} \sum_{l=1}^{M} \sin (k l)(i)^{l-1} \Gamma_{2 l-1 \uparrow},
$$

while for even $j$

$$
\Gamma_{k \uparrow}^{e}=\sqrt{\frac{2}{M+1}} \sum_{l=1}^{M} \sin (k l)(i)^{l-1} \Gamma_{2 l \uparrow} .
$$

The relations for spin-down operators can be obtained by replacing $\uparrow$ with $\downarrow$ and the imaginary unit $i$ with $-i$.

For a finite chain of an even number of sites $L$, the zeroenergy end modes $a_{1 \sigma}, b_{L \sigma}$, are also split by a perturbative process of order $t^{L / 2}$ that involves $\Gamma_{j \sigma}$ and $\Gamma_{j \sigma}^{\dagger}$ with even $j$, as can be seen from Eqs. (31). For odd $L, H_{t}$ mixes $b_{L \sigma}$ with the subspace of $\Gamma_{j \sigma}$ and $\Gamma_{j \sigma}^{\dagger}$ with odd $j$, and $b_{L \sigma}$ remains decoupled from $a_{1 \sigma}$ at finite $t$.

To calculate the perturbative effective coupling between the end modes it is easier to map the problem introducing kets associated with the annihilation $(a)$ and creation $(c)$ operators,

$$
c_{\alpha} \leftrightarrow|\alpha a\rangle, c_{\alpha}^{\dagger} \leftrightarrow|\alpha c\rangle,
$$

and introduce the Hamiltonian

$$
\begin{aligned}
\tilde{H}= & \sum_{\beta \alpha} A_{\beta \alpha}|\beta a\rangle\left\langle\alpha a\left|+B_{\beta \alpha}\right| \beta c\right\rangle\langle\alpha a| \\
& -\bar{A}_{\beta \alpha}|\beta c\rangle\left\langle\alpha c\left|+\bar{B}_{\beta \alpha}\right| \beta a\right\rangle\langle\alpha c|,
\end{aligned}
$$

with coefficients defined by the equations

$$
\left[c_{\alpha}, H\right]=\sum_{\beta}\left(A_{\beta \alpha} c_{\beta}+B_{\beta \alpha} c_{\beta}^{\dagger}\right)
$$

so that solving $\tilde{H}\left|\Gamma_{\nu} a\right\rangle=E_{\nu}\left|\Gamma_{\nu} a\right\rangle$ is equivalent to solving Eq. (3).

In this new language the effective perturbative mixing of the end modes for spin up can be written in the form

$$
\tilde{H}_{m}=V\left|b_{L \uparrow} a\right\rangle\left\langle a_{1 \uparrow} a\right|+\text { H.c. },
$$

where

$$
V=\sum \frac{\left\langle b_{L \uparrow} a\left|H_{t}\right| e_{M}\right\rangle\left(\prod_{l=1}^{M-1}\left\langle e_{l+1}\left|H_{t}\right| e_{l}\right\rangle\right)\left\langle e_{1}\left|H_{t}\right| a_{1 \uparrow} a\right\rangle}{\prod_{l=1}^{M}\left(-E_{l}\right)}
$$

and $\left|e_{l}\right\rangle$ and $E_{l}$ label the two possible intermediate states at each of the $M=L / 2-1$ sites with even $j$ and the corresponding energies. The sum runs over all possible $2^{M}$ combinations of intermediate states. The state $\left|e_{l}\right\rangle$ is either $\left|\Gamma_{2 l \uparrow} a\right\rangle$ with energy $E_{l}=2$ or $\left|\Gamma_{2 l \downarrow} c\right\rangle$ with energy $E_{l}=-2$. Using Eq. (31), it is easy to see that the contribution of the sum when all intermediate states correspond to annihilation operators $\left(\left|\Gamma_{2 l \uparrow} a\right\rangle\right)$ is $-t(-i t / 4)^{M}$. In addition, each time $\left|\Gamma_{2 l \uparrow} a\right\rangle$ is replaced by $\left|\Gamma_{2 l \downarrow} c\right\rangle$, a factor $(-i)^{2}$ appears because of the change in two matrix elements which is compensated by a change in sign in $E_{l}$. Therefore, the $2^{M}$ possibilities of choosing the intermediate states lead to the same contribution. Thus,

$$
\begin{aligned}
& V=-E e^{i \theta}, \\
& E=t(t / 2)^{M}, \\
& e^{i \theta}=(-i)^{M} .
\end{aligned}
$$

The eigenstate of Eq. (40) with positive energy $E$ is $\left(\left|a_{1 \uparrow} a\right\rangle+\right.$ $\left.e^{i \theta}\left|b_{L \uparrow} a\right\rangle\right) / \sqrt{2}$, which corresponds to the annihilation operator

$$
\gamma_{\uparrow}=\frac{1}{\sqrt{2}}\left(a_{1 \uparrow}+e^{i \theta} b_{L \uparrow}\right) .
$$

From time-reversal symmetry, one has for the spin down

$$
\gamma_{\downarrow}=\frac{1}{\sqrt{2}}\left(a_{1 \downarrow}+e^{-i \theta} b_{L \downarrow}\right) .
$$

These results agree with previous ones obtained for a long chain with $\mu=0$ but otherwise arbitrary parameters using an algebraic approach [33]. Here, the nature of the coupling between the end modes becomes more transparent. 


\section{B. Effect of a magnetic field at one end}

Since the total spin projection in the direction of the spinorbit coupling $S_{z}$ is a good quantum number, the effect of a uniform magnetic field in the $z$ direction is trivial and does not modify the eigenstates, just changing the energies. Instead, a magnetic field applied to only one end of the chain leads to nontrivial results. It is easy to generalize Eqs. (45) and (46) to this case, adding to the Hamiltonian the term $-\Delta_{Z} S_{\text {right }}^{z}$, with $\Delta_{Z}=g \mu_{B} B$ and $S_{\text {right }}^{z}=\sum_{j=L / 2}^{L} S_{j}^{z}$ (the total spin projection on the right half of the chain). In practice, only the terms in the sum within a distance to the end of the chain less than or of the order of the localization length of the zero-energy mode contribute because for other sites the singlet character of the superconductor tends to decrease strongly $\left|S_{j}^{z}\right|$. The expectation value of $S_{j}^{z}$ as a function of lattice site $j$ was studied numerically in Ref. [33].

The result for the annihilation operators and energies is [33]

$$
\begin{array}{ll}
\gamma_{\uparrow}=\frac{1}{\sqrt{2}}\left(\alpha a_{1 \uparrow}+\beta e^{i \theta} b_{L \uparrow}\right), & E_{\uparrow}=r-\frac{\Delta_{Z}}{4}, \\
\gamma_{\downarrow}=\frac{1}{\sqrt{2}}\left(\beta a_{1 \downarrow}+\alpha e^{i \theta} b_{L \downarrow}\right), & E_{\downarrow}=r+\frac{\Delta_{Z}}{4},
\end{array}
$$

with

$$
\begin{aligned}
r & =\sqrt{\left(\Delta_{Z} / 4\right)^{2}+E^{2}}, \\
\alpha^{2} & =\frac{1}{2}+\frac{\Delta_{Z}}{4 r}, \quad \beta^{2}=1-\alpha^{2}, \quad \alpha, \beta>0, \\
E & = \begin{cases}t^{L / 2} / 2^{L / 2-1} & \text { if } L \text { is even, } \\
0 & \text { if } L \text { is odd. }\end{cases}
\end{aligned}
$$

\section{Low-energy many-body eigenstates}

Let us discuss first the case without any magnetic field and odd or infinite $L$, so that the end zero modes are not mixed. In this case, following a reasoning similar to the one that led to Eq. (10), one of the states that are part of the ground state is, for small $t$,

$$
\left|e_{1}\right\rangle_{t}=N_{t}\left(\prod_{k} \Gamma_{k \uparrow}^{e} \Gamma_{k \downarrow}^{e}\right)\left(\prod_{k} \Gamma_{k \uparrow}^{o} \Gamma_{k \downarrow}^{o}\right)|0\rangle,
$$

where the annihilation operators are given above [see Eqs. (34), (35), and (36)]. It is important to note that for small $t$ as we assume the energies corresponding to all these operators are positive [Eqs. (34)]. For each spin, the operators $\Gamma_{k \sigma}^{o}$ and $\Gamma_{k \sigma}^{e}$ are related to the local ones $\Gamma_{j \sigma}$ by a unitary matrix $U_{\sigma}$ with coefficients given by Eqs. (35) and (36), with similar notation for spin down. Using this transformation and Eq. (10), it is easy to see that

$$
\left|e_{1}\right\rangle_{t}=\operatorname{det}\left(U_{\uparrow}\right) \operatorname{det}\left(U_{\downarrow}\right)\left|e_{1}\right\rangle .
$$

Thus, $\left|e_{1}\right\rangle_{t}$ corresponds to the same physical state as $\left|e_{1}\right\rangle$.

In the general case, one of the states that is part of the ground state (nondegenerate for finite even $L$ ) has an even number of particles and is given by

$$
\left|g_{e}\right\rangle=N \gamma_{\uparrow} \gamma_{\downarrow}\left|e_{1}\right\rangle
$$

where $N$ is a normalization factor and $\gamma_{\sigma}$ are given by Eqs. (45) and (46).

The other three low-energy states and their excitation energies with respect to the ground state for small enough $t$ and $B$ are

$$
\begin{aligned}
|l \uparrow\rangle & =\gamma_{\uparrow}^{\dagger}\left|g_{e}\right\rangle, E_{\uparrow}, \\
|l \downarrow\rangle & =\gamma_{\downarrow}^{\dagger}\left|g_{e}\right\rangle, E_{\downarrow}, \\
\left|l_{e}\right\rangle & =\gamma_{\uparrow}^{\dagger} \gamma_{\downarrow}^{\dagger}\left|g_{e}\right\rangle, 2 r .
\end{aligned}
$$

Note that for odd or infinite $L, E=0$, which implies $E_{\uparrow}=0$ and a twofold degeneracy remains in the ground state and in the other two low-energy states. For a finite chain with odd $L$, this degeneracy is broken if $\mu \neq 0$ [33]. In addition, for the two states with an even number of particles, $\left|g_{e}\right\rangle$ and $\left|l_{e}\right\rangle$, the total spin projection $S_{z}=0$, while $S_{z}|l \sigma\rangle=\left(s_{\sigma} / 2\right)|l \sigma\rangle$. Defining $S_{\text {left }}^{z}=S_{z}-S_{\text {right }}^{z}$, using previous results [33] and the vanishing of the trace of $S_{z}$ in the low-energy subspace, one obtains

$$
\begin{aligned}
\left\langle g_{e}\left|S_{\text {right }}^{z}\right| g_{e}\right\rangle & =-\left\langle g_{e}\left|S_{\text {left }}^{z}\right| g_{e}\right\rangle \\
& =-\left\langle l_{e}\left|S_{\text {right }}^{z}\right| l_{e}\right\rangle=\left\langle l_{e}\left|S_{\text {left }}^{z}\right| l_{e}\right\rangle \\
& =\frac{\Delta_{Z}}{4 \sqrt{\left(\Delta_{Z}\right)^{2}+16 E^{2}}}, \\
\left\langle l \uparrow\left|S_{\text {right }}^{z}\right| l \uparrow\right\rangle & =\left\langle l \uparrow\left|S_{\text {left }}^{z}\right| l \uparrow\right\rangle \\
& =-\left\langle l \downarrow\left|S_{\text {right }}^{z}\right| l \downarrow\right\rangle=\left\langle l \downarrow\left|S_{\text {left }}^{z}\right| l \downarrow\right\rangle \\
& =1 / 4 .
\end{aligned}
$$

The analytical results valid for small $t$ and $B$ for the excitation energies, Eqs. (52), and the spin projections for the left or right part of the chain, Eqs. (53), agree very well with our numerical results for a finite chain. In Table I we list some of these numerical results for $L=4$. The numerical excitation energies presented in Table I coincide with the analytical results to the precision of the former. For the spin projection, there is a small discrepancy. For example, for $t=0.04$ and $B=0.002$, the analytical result for $S_{\text {right }}^{z}$ in the ground state is larger by $1.0 \times 10^{-4}$. For the excited state in the even subspace, the discrepancy is near $3 \times 10^{-4}$. The accuracy of the analytical results should increase with the length of the chain since the effective mixing of the end states scales as $t^{L / 2}$ [see Eqs. (40), (42), and (43)].

\section{SUMMARY AND DISCUSSION}

We have found the exact analytical solution, for a particular set of parameters, of a standard model for a finite chain of a time-reversal-invariant topological superconductor. This allowed us to construct the degenerate ground state of the system, which consists of two states with even fermion parity and total spin projection $S_{z}=0$ and two states with odd fermion parity and $S_{z}= \pm 1 / 2$. The latter two states have spin projections $S_{z}= \pm 1 / 4$ at the ends. If a magnetic field is applied to one end of the chain, the former two states are split into two states having expectation values of $1 / 4$ at one end and $-1 / 4$ at the other. In addition, creating a zero mode at 
TABLE I. Numerical results for a chain of $L=4$ sites.

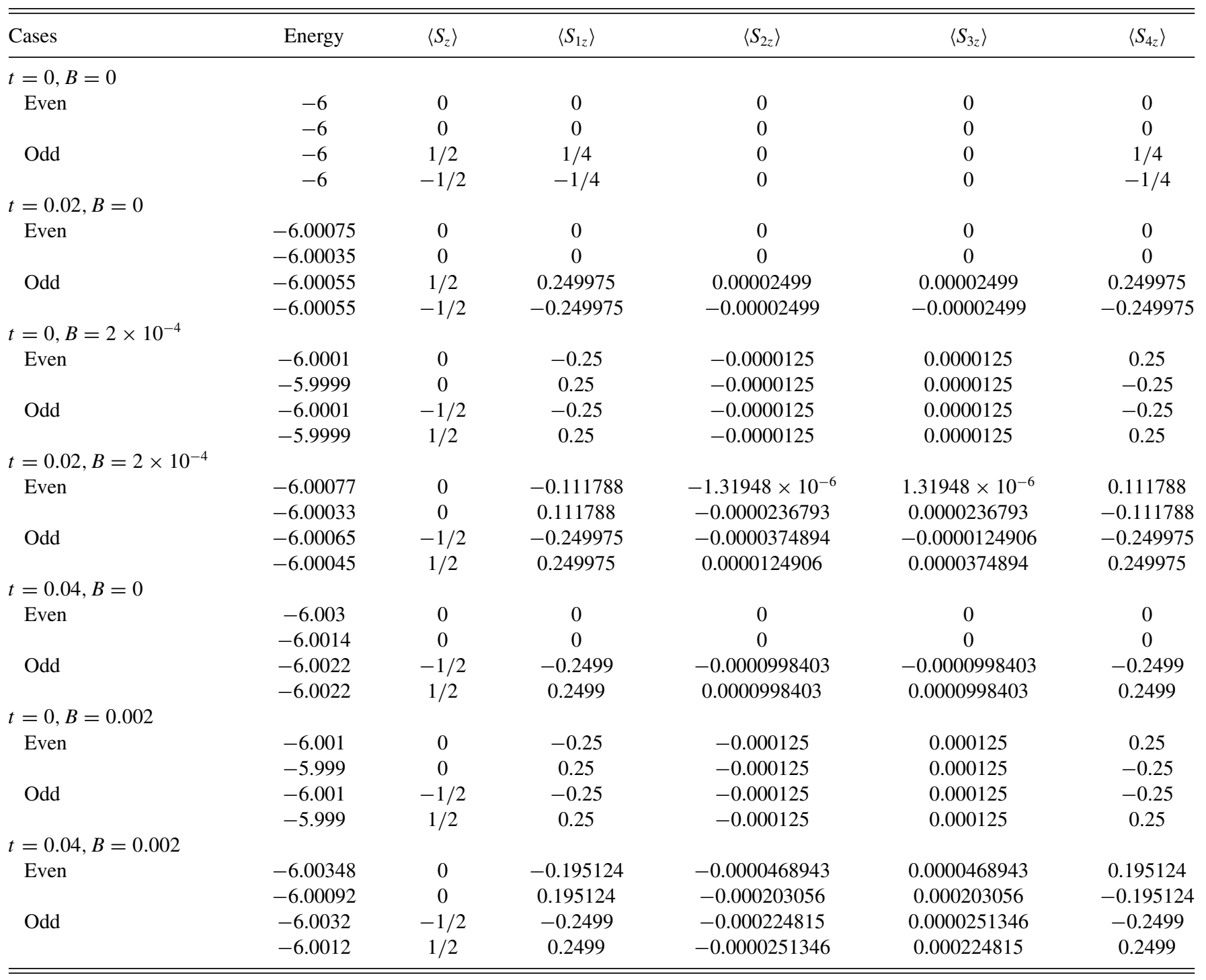

one end or at the other one gives related results. This property might be used for teleportation of Majorana fermions $[43,44]$ or spins [45] since the transport of an electron from one end to the other might be avoided.

Since the ground state is separated from the excited states by a finite gap, by continuity these properties remain true for a chain of infinite length and general values of the parameters. The coefficients of proportionality in some relations [like Eq. (13)] change, but the proportionality itself does not. Specifically, the number of low-energy eigenstates (four) and the values of the good quantum numbers (total spin projection $S_{z}$, total fermion parity $P$, and the related value of $K^{2}$ where $K$ is time reversal) are discrete numbers which should keep their values unmodified under changes of the parameters, unless a phase transition takes place. A previous study of the fractional spin projection at the ends indicated that for general parameters, the localization length of the end modes increases on approaching the nontopological phase and diverges exactly at the transition point [33].
Concerning the discussion on the time-reversal anomaly, in spite of its use to explain different properties of the system [40], some statements should be revised.

The main effect of a finite $t$ (or a finite chain of length $L$ in a general case) is to split the two states of even parity in the ground state by a quantity of order $\exp \left(-L / \lambda_{e}\right)$, where $\lambda_{e}$ is the localization length of the end modes. This localization length increases with increasing $t$. In addition, the absolute value of the fractional expectation values of the spin projection at the ends of the even-parity many-body states under the application of a magnetic field, which is $1 / 4$ for $t=0$, decreases for finite $t$. This might be experimentally detectable [33].

\section{ACKNOWLEDGMENTS}

We thank L. Arrachea for helpful discussions. We acknowledge support from CONICET and UBACyT, Argentina. We are sponsored by Grant No. PIP 112-201501-00506 of CONICET and Grant No. PICT 2017-2726 of ANPCyT. 
[1] A. Kitaev, Fault-tolerant quantum computation by anyons, Ann. Phys. (NY) 303, 2 (2003).

[2] M. H. Freedman, M. Larsen, and Z. Wang, A modular functor which is universal for quantum computation, Commun. Math. Phys. 227, 605 (2002).

[3] A. Y. Kitaev, Unpaired Majorana fermions in quantum wires, Sov. Phys. Usp. 44, 131 (2001).

[4] Y. Oreg, G. Refael, and F. von Oppen, Helical Liquids and Majorana Bound States in Quantum Wires, Phys. Rev. Lett. 105, 177002 (2010).

[5] R. M. Lutchyn, J. Sau, and S. Das Sarma, Majorana Fermions and a Topological Phase Transition in SemiconductorSuperconductor Heterostructures, Phys. Rev. Lett. 105, 077001 (2010)

[6] L. Fu and C. L. Kane, Josephson current and noise at a superconductor/quantum-spin-Hall-insulator/superconductor junction, Phys. Rev. B 79, 161408(R) (2009).

[7] S. Nadj-Perge, I. K. Drozdov, J. Li, H. Chen, S. Jeon, J. Seo, A. H. MacDonald, B. A. Bernevig, and A. Yazdani, Topological matter. Observation of Majorana fermions in ferromagnetic atomic chains on a superconductor, Science 346, 602 (2014).

[8] V. Mourik, K. Zuo, S. M. Frolov, S. R. Plissard, E. P. A. M. Bakkers, and L. P. Kouwenhoven, Signatures of Majorana fermions in in hybrid superconductor-semiconductor nanowire devices, Science 336, 1003 (2012).

[9] A. Das, Y. Ronen, Y. Most, Y. Oreg, M. Heiblum, and H. Shtrikman, Zero-bias peaks and splitting in an Al-InAs nanowire topological superconductor as a signature of Majorana fermions, Nat. Phys. 8, 887 (2012).

[10] S. M. Albrecht, A. P. Higginbotham, M. Madsen, F. Kuemmeth, T. S. Jespersen, J. Nyg, P. Krogstrup, and C. M. Marcus, Exponential protection of zero modes in Majorana islands, Nature (London) 531, 206 (2016).

[11] M. Deng, S. Vaitiekenas, E. Hansen, J. Danon, M. Leijnse, K. Flensberg, J. Nygard, P. Krogstrup, and C. Marcus, Majorana bound state in a coupled quantum-dot hybrid-nanowire system, Science 354, 1557 (2016).

[12] H. J. Suominen, M. Kjaergaard, A. R. Hamilton, J. Shabani, C. J. Palmstrøm, C. M. Marcus, and F. Nichele, Zero-Energy Modes from Coalescing Andreev States in a Two-Dimensional Semiconductor-Superconductor Hybrid Platform, Phys. Rev. Lett. 119, 176805 (2017).

[13] X.-L. Qi, T. L. Hughes, S. Raghu, and S.-C. Zhang, TimeReversal-Invariant Topological Superconductors and Superfluids in Two and Three Dimensions, Phys. Rev. Lett. 102, 187001 (2009).

[14] X.-L. Qi, T. L. Hughes, and S.-C. Zhang, Topological invariants for the Fermi surface of a time-reversal-invariant superconductor, Phys. Rev. B 81, 134508 (2010).

[15] S. Deng, L. Viola, and G. Ortiz, Majorana Modes in TimeReversal Invariant $s$-Wave Topological Superconductors, Phys. Rev. Lett. 108, 036803 (2012).

[16] S. Nakosai, J. K. Budich, Y. Tanaka, B. Trauzettel, and N. Nagaosa, Majorana Bound States and Nonlocal Spin Correlations in a Quantum Wire on an Unconventional Superconductor, Phys. Rev. Lett. 110, 117002 (2013).

[17] S. B. Chung, J. Horowitz, and X.-L. Qi, Time-reversal anomaly and Josephson effect in time-reversal-invariant topological superconductors, Phys. Rev. B 88, 214514 (2013).
[18] F. Zhang, C. L. Kane, and E. J. Mele, Time-Reversal-Invariant Topological Superconductivity and Majorana Kramers Pairs, Phys. Rev. Lett. 111, 056402 (2013).

[19] A. Keselman, L. Fu, A. Stern, and E. Berg, Inducing TimeReversal-Invariant Topological Superconductivity and Fermion Parity Pumping in Quantum Wires, Phys. Rev. Lett. 111, 116402 (2013).

[20] E. Dumitrescu and S. Tewari, Topological properties of time-reversal-symmetric Kitaev chain and applications to organic superconductors, Phys. Rev. B 88, 220505(R) (2013).

[21] A. Haim, A. Keselman, E. Berg, and Y. Oreg, Time-reversalinvariant topological superconductivity induced by repulsive interactions in quantum wires, Phys. Rev. B 89, 220504(R) (2014).

[22] J. Klinovaja and D. Loss, Time-reversal invariant parafermions in interacting Rashba nanowires, Phys. Rev. B 90, 045118 (2014)

[23] J. Klinovaja, A. Yacoby, and D. Loss, Kramers pairs of Majorana fermions and parafermions in fractional topological insulators, Phys. Rev. B 90, 155447 (2014).

[24] M. S. Scheurer and J. Schmalian, Topological superconductivity and unconventional pairing in oxide interfaces, Nat. Commun. 6, 6005 (2015).

[25] C. Schrade, A. A. Zyuzin, J. Klinovaja, and D. Loss, ProximityInduced Josephson $\pi$-Junctions in Topological Insulators, Phys. Rev. Lett. 115, 237001 (2015)

[26] A. Haim, K. Wölms, E. Berg, Y. Oreg, and K. Flensberg, Interaction-driven topological superconductivity in one dimension, Phys. Rev. B 94, 115124 (2016).

[27] J. Li, W. Pan, B. A. Bernevig, and R. M. Lutchyn, Detection of Majorana Kramers Pairs using a Quantum Point Contact, Phys. Rev. Lett. 117, 046804 (2016).

[28] E. Mellars and B. Béri, Signatures of time-reversal-invariant topological superconductivity in the Josephson effect, Phys. Rev. B 94, 174508 (2016).

[29] W.-J. Gong, Z. Gao, W.-F. Shan, and G.-Y. Yi, Influence of an embedded quantum dot on the Josephson effect in the topological superconducting junction with Majorana doublets, Sci. Rep. 6, 23033 (2016).

[30] A. Camjayi, L. Arrachea, A. Aligia, and F. von Oppen, Fractional Spin and Josephson Effect in Time-Reversal-Invariant Topological Superconductors, Phys. Rev. Lett. 119, 046801 (2017).

[31] Ch. Reeg, C. Schrade, J. Klinovaja, and D. Loss, DIII topological superconductivity with emergent time-reversal symmetry, Phys. Rev. B 96, 161407(R) (2017).

[32] F. Parhizgar and A. M. Black-Schaffer, Highly tunable timereversal-invariant topological superconductivity in topological insulator thin films, Sci. Rep. 7, 9817 (2017).

[33] A. A. Aligia and L. Arrachea, Entangled end states with fractionalized spin projection in a time-reversal-invariant topological superconducting wire, Phys. Rev. B 98, 174507 (2018).

[34] C. Schrade and L. Fu, Parity-Controlled $2 \pi$ Josephson Effect Mediated by Majorana Kramers Pairs, Phys. Rev. Lett. 120, 267002 (2018).

[35] L. Lauke, M. S. Scheurer, A. Poenicke, and J. Schmalian, Friedel oscillations and Majorana zero modes in inhomogeneous superconductors, Phys. Rev. B 98, 134502 (2018). 
[36] H. Hu, F. Zhang, and C. Zhang, Majorana Doublets, Flat Bands, and Dirac Nodes in $s$-Wave Superfluids, Phys. Rev. Lett. 121, 185302 (2018).

[37] M. Mashkoori, A. G. Moghaddam, M. H. Hajibabaee, A. M. Black-Schaffer, and F. Parhizgar, Impact of topology on the impurity effects in extended $s$-wave superconductors with spin-orbit coupling, Phys. Rev. B 99, 014508 (2019).

[38] O. E. Casas, L. Arrachea, W. J. Herrera, and A. Levy Yeyati, Proximity induced time-reversal topological superconductivity in $\mathrm{Bi}_{2} \mathrm{Se}_{3}$ films without phase tuning, Phys. Rev. B 99, 161301(R) (2019).

[39] L. Arrachea, A. Camjayi, A. A. Aligia, and L. Gruñeiro, Catalog of Andreev spectra and Josephson effects in structures with time-reversal-invariant topological superconductor wires, Phys. Rev. B 99, 085431 (2019).
[40] A. Haim and Y. Oreg, Time-reversal-invariant topological superconductivity, arXiv:1809.06863.

[41] C. Schrade and L. Fu, Quantum Computing with Majorana Kramers Pairs, arXiv:1807.06620.

[42] A. P. Schnyder, S. Ryu, A. Furusaki, and A. W. W. Ludwig, Classification of topological insulators and superconductors in three spatial dimensions, Phys. Rev. B 78, 195125 (2008), and references therein.

[43] L. Arrachea (private communication).

[44] L. Fu, Electron Teleportation via Majorana Bound States in a Mesoscopic Superconductor, Phys. Rev. Lett. 104, 056402 (2010).

[45] R. L. de Visser and M. Blaauboer, Deterministic Teleportation of Electrons in a Quantum Dot Nanostructure, Phys. Rev. Lett. 96, 246801 (2006); Erratum: Deterministic Teleportation of Electrons in a Quantum Dot Nanostructure [Phys. Rev. Lett. 96, 246801 (2006)], 97, 069901 (2006). 